\title{
Response-compatibility effects in focused-attention tasks: A same-hand advantage in response activation
}

\author{
JEFF MILLER \\ University of California, San Diego, La Jolla, California
}

\begin{abstract}
Previous studies have shown that responses can be partially activated by irrelevant stimuli in focused-attention tasks. In two experiments, such response activation was used to investigate the organization of keypress responses. Stimuli were rows of three letters, with a relevant target letter in the middle of the row and irrelevant flanker letters on the outside. There were four target letters, and these were assigned to four keypress responses made with the index and middle fingers of the two hands. Irrelevant flankers were parentheses, a neutral letter, or one of the four target letters. Responses were fastest when flankers were identical to the target, indicating facilitation of a response activated by both relevant and irrelevant letters. When flankers were response-incompatible target letters, responses were faster if these were target letters assigned to a response finger on the same hand as the correct response than if they were target letters assigned to a response finger on the opposite hand. The latter result is consistent with the hypothesis that simultaneous activation of two response fingers on the same hand produces faster responses than simultaneous activation of two response fingers on different hands, as assumed by Miller (1982). In Experiment 2, flankers were presented slightly before targets, and flankers that were same-hand target letters sometimes facilitated responses relative to the neutral parentheses flankers.
\end{abstract}

In a series of papers concerning visual selective attention, C. W. Eriksen and his coworkers have documented a sizable response-compatibility effect of unattended stimuli. In one series of experiments, for example, subjects were presented with rows of letters and told to respond to the identity of the middle letter, ignoring the flanker letters on either side (C. W. Eriksen \& Schultz, 1979). Even though the flankers were to be ignored, responses were faster when flankers were targets assigned to the same response as the relevant middle letter (response-compatible trials) than when flankers were target letters assigned to the opposite response (responseincompatible trials). Reaction time (RT) was intermediate when the flankers were response-neutral letters, in dicating that target flankers produced both facilitation and inhibition.

Two recent studies indicate that much or all of this response-compatibility effect is produced by competition in response activation processes. C. W. Eriksen, Coles, Morris, and O'Hara (1985) measured electromyographic (EMG) activity as an index of response activation. They found that response-incompatible displays caused more

This research was supported by NIMH Grant PHS-MH40733. I would like to thank C. W. Eriksen, Patricia Haden, Roland Schaffer, and two anonymous reviewers for helpful comments on earlier drafts of the paper, particularly suggestions leading to Experiment 2 . I would also like to thank Grace Fang, Alann Lopes, Brent Roberts, and Thomica James for assistance in collecting the data and in preparing the figures. Re quests for reprints should be addressed to the author at the Department of Psychology, C-009, UCSD, La Jolla, CA 92093.
EMG activity in the incorrect response arm than did response-compatible displays, indicating that flankers "were processed to the point of incipient response activation" (C. W. Eriksen et al., 1985, p. 165). For both compatible and incompatible displays, responses were slower on trials with observable EMG activity on the incorrect side than on trials without it, so the response activation produced by incompatible flankers seems to have been instrumental in slowing responses.

Coles, Gratton, Bashore, C. W. Eriksen, and Donchin (1985) extended these findings with a measure of response activation sensitive to even more peripheral motor processes. In their experiment, subjects responded by squeezing dynamometers and partial responses could be observed directly (i.e., squeezes less than the criterion for a response). Response-incompatible displays produced morc partial squeezes with the incorrect response hand than did response-compatible displays, indicating that response-incompatible flankers produce not only incipient response activation, but also some actual motor activity. As expected, correct responses were slower on trials with partial squeezes on the incorrect side than on trials without such partial squeezes. EMG was also included as a measure of response activation, and effects on EMG were parallel to those of C. W. Eriksen et al. (1985) discussed above. Interestingly, the interval from the onset of EMG activity on the correct side to the onset of squeeze activity on that same side was lengthened if there was squeeze activity on the incorrect side. This indicates that inappropriate response activation not only delayed the onset 
of correct response activation, but also reduced the rate at which it built up to the threshold needed for motor activity (see also Coles \& Gratton, 1986).

If the flanker compatibility effect arises during response activation, then this effect may be useful in studying the organization of the motor system. It is commonly believed that response movements are represented internally by a set of defining motor features, and a number of investigators have suggested methods of identifying these features (e.g., Rosenbaum, 1980; Rosenbaum \& Kornblum, 1982). Most of the methods involve cuing a subject to get ready for one or two particular responses out of a larger response set, but cuing introduces the possibility of significant strategic effects (Rosenbaum, 1983; Rosenbaum \& Kornblum, 1982). Thus, a converging methodology would be valuable, especially if it did not involve explicit cuing.

The flanker-compatibility effect may provide an alternative methodology to investigate the defining characteristics of movements, if-as the evidence indicates-it reflects response activation. Specifically, the RT for a given movement can be examined to see if it varies according to what other movement is partially activated at the same time by the flankers. To the extent that the other movement is dissimilar, competition between the two simultaneously activated responses should be maximal and responses should be slow (e.g., Berlyne, 1957; Heuer, 1982; Rosenbaum, Inhoff, \& Gordon, 1984; but see Buckolz, 1982). To the extent that the other movement is similar, competition should be minimal and responses should be fast.

The present experiments used the flanker-compatibility effect to investigate featural similarities among keypress responses in a four-choice task, with the four keypress responses made by the index and middle fingers of the right and left hands. Three types of response similarity were compared. First, the flankers could activate the response on the same hand as the correct response, so that the two activated movements shared the hand feature. Second, the flankers could activate the response on the same finger as the correct response, but on the opposite hand, so that the two activated movements shared the finger feature (i.e., were homologous). Third, the flankers could be letters assigned to the response key on the nonhomologous finger on the other hand (neither same) from the response key assigned to the actual target letter.

Two other types of flankers were also included for comparison purposes. Neutral flankers (i.e., flankers not assigned to any response) were tested to get an estimate of baseline RT unaffected by response activation from the flankers. Response-compatible flankers (i.e., flankers identical to the center target) were also included to estimate the facilitation produced when flankers activated the correct response.

This set of keypress responses was chosen for study mainly to test an assumption of a paradigm proposed by Miller (1982), which used this same four-finger response set, although it is also of interest because the organization of fine digital responses is inherently important for many higher skilled tasks, including typing and piano playing. The hand-mapping paradigm (Miller, 1982) was suggested as a way of determining whether response preparation could begin before stimulus recognition was finished, as predicted by continuous models of human information processing (e.g., C. W. Eriksen \& Schultz, 1979; McClelland, 1979) and prohibited by discrete models (e.g., Sternberg, 1969). Miller (1982) assumed that responses would be faster when partial stimulus information activated two response fingers on the same hand than when it activated two response fingers on different hands. The assumed hand-preparation advantage was obtained in cuing experiments, but recent evidence has raised questions about the validity of such experiments for assessing response activation. In particular, Reeve and Proctor $(1984,1985)$ have argued that the hand-preparation advantage in cuing experiments is actually caused by an interaction of S-R translation processes with the nature of the cue (but see Miller, 1985, 1987). In view of the debate about the interpretation of the hand-preparation advantage obtained in cuing experiments, it seemed worthwhile to conduct further tests of the assumption that this advantage reflects response activation.

\section{EXPERIMENT 1}

\section{Method}

Subjects and Apparatus. Forty-two undergraduates at the University of California, San Diego, served as subjects in partial fulfillment of a course requirement. Each subject was tested in a single session lasting about $45 \mathrm{~min}$. Stimuli were presented and responses and their latencies recorded by an Apple II+ microcomputer. The outside four keys on the bottom row of the computer keyboard were used as response keys, and subjects responded by pressing these keys with the index and middle fingers of the left and right hands.

Stimuli. Stimuli were uppercase consonants presented in the standard font of the computer. Letters appeared as light green figures on the dark background of the computer terminal, and each letter was approximately $4.8 \mathrm{~mm}$ high and $3.2 \mathrm{~mm}$ wide. A stimulus display was a row of three such letters, with a center-to-center separation of $10 \mathrm{~mm}$ between letters. Viewed from a distance of about $60 \mathrm{~cm}$, the row of letters subtended about $1.25^{\circ}$ of visual angle. The outside two letters of each row were always identical to each other.

Procedure. For each subject, five letters were randomly selected from the set of uppercase consonants. Four of these were assigned, in random order, to the four response keys. The fifth was used as a neutral flanker.

Each subject completed three blocks of trials, identical except for the randomization of trial order. Within each block there were 20 types of trials, defined by four possible target letters (i.e., four possible responses) times five possible flanker letters (the four target letters plus one neutral letter). Each type of trial was tested nine times within each block.

The subjects were instructed that they would be presented with rows of three letters and that they should respond to the middle letter, ignoring the two letters on the outside. The mapping of target letters to response keys was given, and subjects were encouraged to respond as quickly as possible without making too many errors.

Each trial began with the presentation, for $500 \mathrm{msec}$, of a plus sign that served as both a fixation point and a warning signal. One second after the offset of the plus sign, a row of three letters ap- 
peared, and it remained until the subject responded. Accuracy feedback was given after each response $(600 \mathrm{msec}$ for correct responses and $1.2 \mathrm{sec}$ for errors), and there was a delay of approximately $1 \mathrm{sec}$ from the offset of the accuracy feedback to the onset of the plus sign beginning the next trial.

\section{Results and Discussion}

Data from 1 subject were discarded because of nearrandom responding. For the other subjects, mean $\mathrm{RT}$ and percentage of correct responses (PC) were computed for each condition within each block of trials. Figure 1 shows RT, averaged across subjects, blocks, and responses, as a function of flanker type.

Statistical significance was evaluated with an overall analysis of variance (ANOVA) using the factors block, response, and flanker type. All three factors produced significant effects on RT. The main effect of flanker type $[F(4,160)=23, p<.01, M S \mathrm{e}=10,548]$ reflects the differences among conditions shown in Figure 1. Post hoc comparisons using the Newman-Keuls test indicated that the three-tiered pattern of conditions apparent in Figure 1 was significant $(p<.01)$. Responses were significantly faster with identical flankers than with any of the other types of flankers; responses were significantly faster with neutral or same-hand flankers than with same-finger or neither-same flankers.

The main effect of block was also significant in the overall ANOVA of RT $[F(2,80)=3.39, p<.05, M S \mathrm{e}=$ 72,824]. Overall RTs decreased monotonically across the three blocks, with a difference of $35 \mathrm{msec}$ from the first block to the last, presumably due to practice. The effect of response was significant $[F(3,120)=6.36, p<.01$, $M S \mathrm{e}=75,905]$, with RTs of $888 \mathrm{msec}$ for the left middle finger, $950 \mathrm{msec}$ for the left index finger, $945 \mathrm{msec}$ for the right index finger, and $926 \mathrm{msec}$ for the right middle finger. Pairwise comparisons using the Newman-Keuls test indicate that responses with the left middle finger were significantly faster than those with either of the two in- dex fingers, and that none of the other pairwise differences were significant. The advantage for the left middle finger may stem from a left-to-right strategy for rehearsing the assignments of letters to response keys.

Overall response accuracy was relatively good, with an average PC of about $96 \%$. PC did not depend on flanker type $[F(4,160)=1.56, p>.15, M S e=43.78]$. Subjects often make more than $4 \%$ errors in two-choice versions of this task, especially with response-incompatible flankers (e.g., C. W. Eriksen \& Schultz, 1979; but see Flowers \& Wilcox, 1982, or Grice, Boroughs, \& Canham, 1984 , for other studies with low error rates). Perhaps subjects adopted a more stringent response criterion in this four-choice task than they usually do in two-choice tasks, and this stringent criterion reduced both the overall error rate and the effect of flankers on error rate.

This experiment demonstrates the existence of response-compatibility effects in a four-choice version of the focused-attention task studied by C. W. Eriksen and his colleagues (e.g., C. W. Eriksen \& Schultz, 1979). Response-compatible flankers produce faster responses than response-incompatible flankers, just as they do in two-choice tasks.

The use of a four-choice task led to the additional finding that, when flankers are response incompatible, RT depends on the relationship between the actual response and the other response activated by the flankers. With this response set, responses were faster when the flanker activated a finger on the same hand as the correct response than when it activated a finger on the other hand. In fact, responses on trials with same-hand flankers were just as fast as responses with neutral flankers. There was no advantage for the homologous finger on the nonresponding hand as opposed to the nonhomologous finger on that hand.

These results are quite consistent with the view, derived from cuing studies (Miller, 1982, 1985), that the handpreparation advantage is due to response-activation pro-

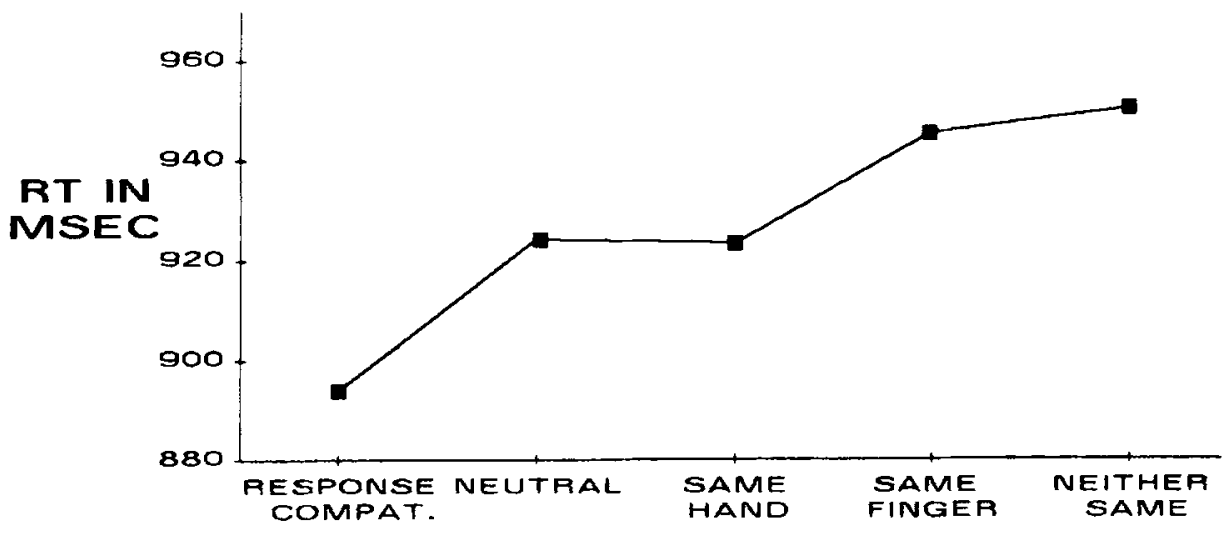

FLANKER TYPE

Figure 1. Experiment 1. Reaction time to a center target letter as a function of the response to which the irrelevant flanker letters were assigned. 
cesses. Since previous studies have provided strong evidence that flanker-compatibility effects reflect response competition (Coles et al., 1985; C. W. Eriksen et al., 1985), it seems quite natural also to attribute the present advantage for same-hand over different-hand flankers to response processes as well. Specifically, the present results can be explained by assuming that simultaneous activation of two responses made by fingers on different hands leads to more interference than simultaneous activation of two responses on the same hand. Thus, the present findings are entirely consistent with the assumption made by Miller (1982) in developing a paradigm to discriminate between discrete and continuous models.

Since same-hand flankers produced responses just as fast as neutral flankers, it appears that these responseincompatible flankers produced no net response competition at all. Given previous evidence that responseincompatible flankers do produce response competition, the most likely explanation of this zero net effect is that there is a source of facilitation when two fingers on the same hand are activated simultaneously. This facilitation would cancel out the response competition, resulting in no net effect of same-hand flankers. Such facilitation is not necessary for the assumption made by Miller (1982), but would be interesting to demonstrate in its own right.

\section{EXPERIMENT 2}

If the response activation produced by same-hand flankers actually facilitates responses, it might be possible to set up conditions under which responses would be faster with such flankers than with neutral flankers. Such a finding would be direct evidence of the facilitatory effects of same-hand response activation, whereas the finding of an advantage of same-hand over different-hand flankers in Experiment 1 is only indirect evidence of facilitation.

In previous research using unattended flankers, facilitatory effects of response-compatible flankers have been largest when the flankers were presented slightly before the target (e.g., Flowers \& Wilcox, 1982; Grice et al., 1984). This suggests that facilitatory effects of responseincompatible, same-hand flankers might also be larger if flankers were presented somewhat before the target. To optimize same-hand facilitation in the present experiment, then, flankers were presented with stimulus onset asynchronies (SOAs) of 50,100, or $150 \mathrm{msec}$ before target onset.

To demonstrate facilitatory effects of same-hand flankers, it is necessary to have an appropriate neutral condition. This experiment used the neutral condition suggested by Grice et al. (1984)-parentheses surrounding the target letter. Parentheses are better than a no-flanker condition, because they provide the same temporal cuing as other flankers and they control for the presence of a visual form on each side of the target.

As a control to test for facilitatory effects of same-hand flankers on response activation, the parentheses neutral condition is somewhat conservative. Since the parentheses are easily discernible as nonletters, they should produce much less perceptual processing load or distraction than a nontarget neutral condition like that used in Experiment 1 . Same-hand flankers could facilitate responding and yet not produce faster overall RTs than parentheses if they slowed perceptual processing. If facilitation were to be observed in such a conservative comparison, of course, the results would be all the more convincing that the facilitation was real.

\section{Method}

The subjects were 150 undergraduates from the same pool tested in Experiment 1. Given the error variance obtained in the previous experiment, this many subjects were needed to have adequate power to detect the small advantage (roughly $10 \mathrm{msec}$ ) that might be expected for same-hand over neutral flankers. The apparatus and procedure were the same as those used in the previous experiment, with two exceptions. First, the flankers appeared 50, 100, or $150 \mathrm{msec}$ before the center target. Second, the neutral flankers were parentheses. Each combination of flanker type and SOA was tested three times in each block.

\section{Results and Discussion}

Figure 2 shows RT as a function of flanker type, averaged across subjects, blocks, responses, and SOA. As in Experiment 1, responses were fastest when flankers were identical to targets and slowest when flankers were targets assigned to responses on the opposite hand. As predicted by the hypothesis that same-hand flankers can sometimes facilitate response activation, responses were 9 msec faster with same-hand than with neutral flankers.

Overall statistical analyses of RT and PC similar to those of Experiment 1 were conducted, with the additional factor of SOA. The effect of flanker type on RT, shown in Figure 2, was highly significant $[F(4,596)=62$, $p<.01, M S e=32,544]$. Post hoc comparisons using the Newman-Keuls test indicated that there were significant $(p<.02)$ differences between mean RTs for all pairs of conditions except same-finger and neither-same. Thus, even the 9-msec advantage of same-hand over neutral flankers was statistically reliable. Flanker type did not significantly affect $\mathrm{PC}[F(4,596)=1.6, p>.15]$. Accuracy ranged from $96.6 \%$ to $97.0 \%$ across the five flanker types, and accuracies were identical for same-hand and neutral flankers $(96.9 \%)$. Thus, it appears that a response-incompatible flanker can actually facilitate the response, if it activates a finger on the same hand as the response that is to be made. This finding suggests that there may be facilitatory interactions between processes activating different response fingers on the same hand.

A number of other sources of variance were significant in the analysis of RT. The main effect of response was significant $[F(3,447)=30, p<.01, M S \mathrm{e}=255,297]$, with the same ordering of response averages found in Experiment 1 . RT also decreased slightly as SOA increased $[F(2,298)=35, p<.01, M S \mathrm{e}=23,800]$, probably due to an alerting function of the flankers. RT also decreased with successive blocks of practice $[F(2,298)=40, p<$ 


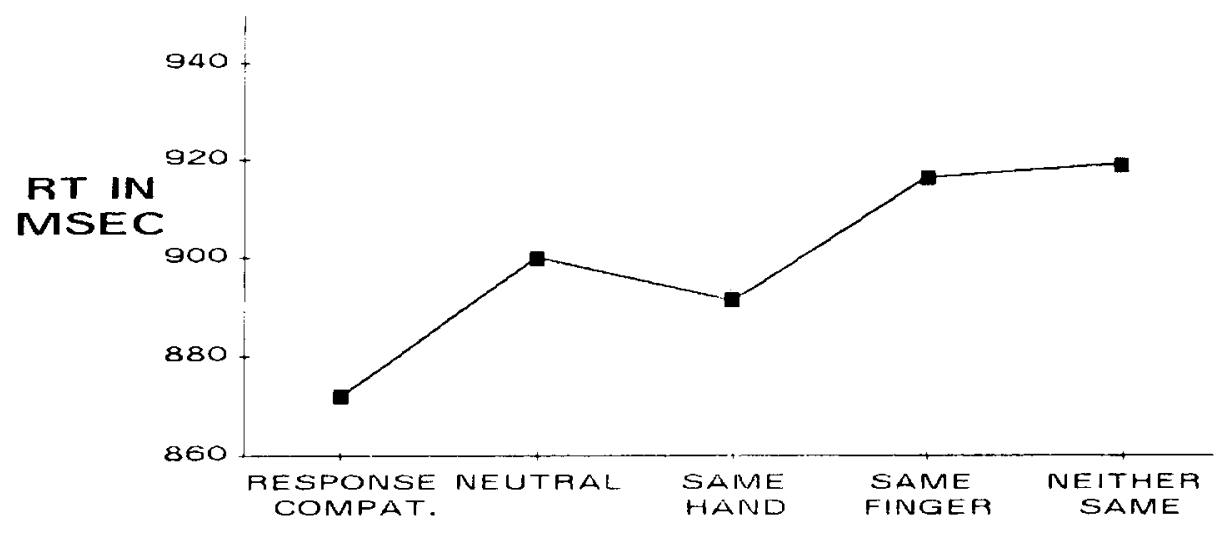

FLANKER TYPE

Figure 2. Experiment 2. Reaction time to a center target letter as a function of the response to which the irrelevant flanker letters were assigned.

$.01, M S e=154,494]$, especially from the first to the second block. There was also an interaction of flanker type and response $[F(12,1788)=5.7, p<.01, M S e=$ 24,044]. The overall effect of flanker type (neither-same minus response-compatible) was much larger for the two middle finger responses (59 and $72 \mathrm{msec}$ for left and right, respectively) than for the two index finger responses (29 and $31 \mathrm{msec}$ ). The advantage for same-hand over neutral flankers was smaller for the right middle finger $(-4 \mathrm{msec})$ than for the other three fingers $(15,10$, and $14 \mathrm{msec}$ ). The interaction of response and block was also significant $[F(6,894)=4.8, p<.01, M S e=37,136]$ due to a much smaller practice effect on the left middle finger $(24 \mathrm{msec})$ than on any of the others $(54,51$, and $63 \mathrm{msec}$ ).

More relevant to the present concerns was a significant three-way interaction of flanker type, SOA, and block $[F(16,2384)=1.88, p<.02, M S e=23,848]$. Inspection of the mean RTs suggested that this interaction was entirely due to the same-hand flanker condition; in fact, the three-way interaction did not approach significance when this condition was omitted from the analysis $(p>.5)$. In view of this significant interaction involving same-hand flankers, it seemed necessary to examine in detail the advantage for same-hand over neutral flankers as a function of block and SOA. Table 1 shows the advantage in mean RT for the same-hand flankers, as well as the statistical significance of each advantage. In each

Table 1

Experiment 2: RT Advantage (in msec) for Same-Hand Over Neutral Flankers as a Function of Block and SOA

\begin{tabular}{ccccc}
\hline & \multicolumn{3}{c}{ SOA } & \\
\cline { 2 - 5 } Block & 50 & 100 & 150 & Average \\
\hline 1 & 16 & 7 & $41^{*}$ & $21^{*}$ \\
2 & 11 & 7 & -9 & 4 \\
3 & 3 & 9 & -6 & 2 \\
Average & $10 \dagger$ & 7 & $9 \dagger$ & $9 \ddagger$ \\
\hline$\dagger p<.10 . \quad \ddagger p<.05$. & ${ }^{*} p<.01$. All other entries, $p>.10$.
\end{tabular}

comparison, statistical significance was evaluated with an ANOVA, using only the conditions with same-hand and neutral flankers and only the block(s) and/or SOA(s) appropriate for each entry in the table.

A large advantage for same-hand over neutral flankers was found in Block 1 at an SOA of $150 \mathrm{msec}$. As indicated in Table 1, this advantage was highly reliable in an analysis of only the data from this condition. It was even highly significant by a sign test, since 98 of the 150 subjects were faster with same-hand than with neutral flankers in this condition.

No other combination of block and SOA showed a clearcut facilitation by same-hand flankers. At the $150-\mathrm{msec}$ SOA, in fact, neutral flankers were responded to slightly, though nonsignificantly, faster than same-hand flankers in the second and third blocks of trials. Averaging across blocks of trials, a marginally significant $(p<.10)$ facilitation was found at the 50 -msec SOA.

\section{GENERAL DISCUSSION}

In two four-choice focused-attention experiments, responses to a center target letter were faster when irrelevant flankers were letters assigned to another response finger on the same hand as the target than when flankers were letters assigned to a response finger on the other hand. Given previous evidence that compatibility effects in this task arise from response-activation processes (Coles et al., 1985; C. W. Eriksen et al., 1985), this finding supports the conclusion that activation of two fingers on the same hand leads to faster responses than activation of two fingers on opposite hands. Thus, the findings are quite consistent with the assumption made by Miller (1982) in comparing discrete and continuous models of human information processing.

One possible explanation for the advantage associated with same-hand rather than opposite-hand flankers is that two movements on the same hand may share defining movement characteristics, so that simultaneous activation 
of both movements leads to less mutual interference than simultaneous activation of two finger responses on different hands (cf. Rosenbaum, 1983). There was virtually no evidence for a finger-based feature in the present results, however. In neither experiment were responses faster when flankers activated the homologous rather than the nonhomologous response finger on the other hand.

Under some conditions in Experiment 2, simultaneous activation of two responses on the same hand actually facilitated responding, as if the mutual activation of the shared-hand feature produces benefit that more than outweighs the cost of competition due to simultaneous activation of different fingers on the hand. In Experiment 2, parentheses were used as neutral flankers, and these may be easier to filter out perceptually than flanker letters, because of their distinctive and non-letter-like shapes. Nonetheless, early in practice, same-hand flankers did produce faster responses than did these neutral flankers at an SOA of $150 \mathrm{msec}$. It is not clear why the advantage for same-hand over neutral flankers should be so volatile, or why this particular combination of practice and SOA should maximize the effect, especially since the overall flanker effect was not largest at the same combination.

Although the present results support the hypothesis that the hand-preparation advantage is due to response activation, as assumed by Miller (1982), they provide no evidence about whether the advantage accrues to two simultaneously activated fingers on the same hand or on the same side of the body. In this experiment, response hand and response side were completely confounded (cf. Reeve $\&$ Proctor, 1984), and it may actually be that the advantage accrues to simultaneous activation of two fingers on the same side rather than the same hand. In this case, the features controlling response activation would correspond to environmental rather than effector organization, so they would have to be considered as premotoric response features. It should be noted, however, that this distinction is not directly relevant to Miller's (1982) paradigm. In that paradigm it was assumed that the hand-preparation advantage was due to response activation, but it was not assumed that the advantage was tied to hand rather than side.

It is difficult to distinguish unambiguously between decision-level and response-level effects on RT. For example, Reeve and Proctor (1984) provided evidence that the hand-preparation advantage in cuing experiments is associated at least partly with response locations rather than response effectors. On the basis of previous evidence that S-R compatibility effects-the standard example of a decision-level effect-belong to locations rather than effectors (but see Klapp, Greim, Mendicino, \& Koenig, 1979), they interpreted this finding as evidence that the advantage arose from decision processes. While not without some plausibility, this interpretation is not necessary. Even if the cuing advantage is associated with loca- tions rather than effectors, it could still arise from response activation rather than from decision processes.

One might also attempt to explain the present results within a decision process, especially since there are few constraints on how such a process must be influenced by unattended stimuli. One possibility is that the decision process occasionally selects a response on the basis of flanker identity at first, and then reselects on the basis of the target. It is possible that the reselection would be faster when the target called for a similar response rather than for a dissimilar one, if the original selection had a residual influence on the state of the decision process. On this view, it is difficult to explain how the decision process manages to reselect rather than just initiate an erroneous response. Worse yet, this view seems to predict that response-incompatible flankers of any type (same- or different-hand) would produce much slower responses than neutral flankers, since neutral flankers would not support an initial, incorrect response selection. In other words, it is especially difficult to see how a decision-level effect could produce same-hand facilitation.

A second possibility is that the decision process chooses a response only to the target, but that it is influenced in some way by the flankers that have not been completely filtered out by selective attention. For some reason, the decision process might find it easier to select a response in the presence of an irrelevant flanker calling for a similar response than in the presence of an irrelevant flanker calling for a dissimilar response. There could even be some decision-level facilitation when the flanker called for a similar response, to explain the facilitation found in Experiment 2. Although such an explanation cannot be ruled out on the basis of the present results, it has four major defects. First, it is little more than a restatement of the data, with no specific decision mechanisms provided to generate the observed effects. Second, it is unparsimonious to attribute the same-hand flanker advantage to the decision process when this advantage can be explained in terms of the response-activation mechanisms already demonstrated by other results (e.g., Coles et al., 1985). Third, decision processes are generally characterized as being severely impaired when forced to process multiple targets (e.g., Duncan, 1980), whether because of limited resources (Navon \& Gopher, 1979, 1980) or because of outcome conflict (Navon, 1984, 1985; Navon \& Miller, 1987). Thus, it is quite implausible that they could produce the same-hand facilitation observed in Experiment 2. Fourth, there is evidence that flankers prime responses directly, without mediation by the active process that selects the response. For example, B. A. Eriksen, C. W. Eriksen, and Hoffman (1986) found additive effects of memory set size and response compatibility, suggesting that irrelevant flankers are filtered out by selective attention before the memory comparison process is invoked. Assuming that the memory comparison process is part of the decision-making process, this indicates that 
irrelevant flankers activate responses by a path that bypasses the deliberate decision process set up by the subject to make explicit response selections.

In conclusion, the response-competition paradigm may be rather generally useful in studying the organization of effector systems. The paradigm seems to reveal the extent of inhibitory interactions between two separate responses that are simultaneously activated, thereby providing clues as to effector organization. It will be interesting to compare results from this paradigm with those of other techniques designed to study the same systems.

\section{REFERENCES}

Berlyne, D. E. (1957). Conflict and choice time. British Journal of Psychology, 48, 106-118.

Buckolz, E. (1982). The influence of the similarity of retrieved response tendencies on the ability to simultaneously initiate and/or suppress outputs. Acta Psychologica, 52, 1-21.

Coles, M. G. H., \& Gratton, G. (1986). Cognitive psychophysiology and the study of states and processes. In G. R. J. Hockey, A. W. K. Gaillard, \& M. G. H. Coles (Eds.), Energetics and human information processing (pp. 409-424). Dordrecht, The Netherlands: Martinus Nijhoff.

Coles, M. G. H., Gratton, G., Bashore, T. R., Eriksen, C. W., \& Donchin, E. (1985). A psychophysical investigation of the continuous flow model of human information processing. Journal of Experimental Psychology: Human Perception \& Performance, 11, 529-553.

DUNCAN, J. (1980). The locus of interference in the perception of simultaneous stimuli. Psychological Review, 87, 272-300.

Eriksen, B. A., Eriksen, C. W., \& HoffMAN, J. E. (1986). Recognition memory and attentional selection: Serial scanning is not enough. Journal of Experimental Psychology: Human Perception \& Performance, 12, 476-483.

Eriksen, C. W., Coles, M. G. H., Morris, L. R., \& O'Hara, W. P. (1985). An electromyographic examination of response competition. Bulletin of the Psychonomic Society, 23, 165-168.

ERIKSEN, C. W., \& SCHULTZ, D. W. (1979). Information processing in visual search: A continuous flow conception and experimental results. Perception \& Psychophysics, 25, 249-263.

FLowers, J. H., \& WiLcox, N. (1982). The effect of flanking context on visual classification: The joint contribution of interactions at different processing levels. Perception \& Psychophysics, 32, 581-591.

Grice, G. R., Boroughs, J. M., \& Canham, L. (1984). Temporal dynamics of associative interference and facilitation produced by visual context. Perception \& Psychophysics, 36, 499-507.

Heuer, H. (1982). Binary choice reaction time as a criterion of motor equivalence. Acta Psychologica, 50, 35-47.

Klapp, S. T., Greim, D. M., Mendicino, C. M., \& Koenig, R. S. (1979). Anatomic and environmental dimensions of stimulus-response compatibility: Implication for theories of memory coding. Acta Psychologica, 43, 367-379.

MCClelland, J. L. (1979). On the time relations of mental processes: A framework for analyzing processes in cascade. Psychological Review, 86, 287-330.

MiLler, J. O. (1982). Discrete versus continuous stage models of human information processing: In search of partial output. Journal of Experimental Psychology: Human Perception \& Performance, 8, 273-296.

Miller, J. O. (1985). A hand advantage in preparation of simple keypress responses: Reply to Reeve and Proctor (1984). Journal of Experimental Psychology: Human Perception \& Performance, 11, 221-233.

Miller, J. O. (1987). Evidence of preliminary response preparation from a divided attention task. Journal of Experimental Psychology: Human Perception \& Performance, 13, 425-434.

Navon, D. (1984). Resources-A theoretical soup stone? Psychological Review, 91, 216-234.

Navon, D. (1985). Attention division or attention sharing? In M. I. Posner \& O. S. M. Marin (Eds.), Attention and performance XI (pp. 133-146). Hillsdale, NJ: Erlbaum.

NAVON, D., \& GoPHER, D. (1979). On the economy of the human information processing system. Psychological Review, 86, 214-255.

NAvoN, D., \& GoPhER, D. (1980). Task difficulty, resources, and dualtask performance. In R. S. Nickerson (Ed.), Attention and performance (Vol. 8, pp. 297-315). Hillsdale, NJ: Erlbaum.

Navon, D., \& Miller, J. O. (1987). Role of outcome conflict in dualtask interference. Joumal of Experimental Psychology: Human Perception \& Performance, 13, 435-448.

ReEve, T. G., \& Proctor, R. W. (1984). On the advance preparation of discrete finger responses. Journal of Experimental Psychology: Human Perception \& Performance, 10, 541-553.

ReEve, T. G., \& Proctor, R. W. (1985). Nonmotoric translation processes in the preparation of discrete finger responses: A rebuttal of Miller's (1985) analysis. Journal of Experimental Psychology: Human Perception \& Performance, 11, 234-241.

Rosenbaum, D. A. (1980). Human movement initiation: Specification of arm, direction, and extent. Journal of Experimental Psychology: General, 109, 444-474.

Rosenbaum, D. A. (1983). The movement precuing technique: Assumptions, applications, and extensions. In R. A. Magill (Ed.), Memory and control of action (pp. 231-274). Amsterdam: North Holland.

Rosenbaum, D. A., Inhoff, A. W., \& Gordon, A. M. (1984). Choosing between movement sequences: A hierarchical editor model. Journal of Experimental Psychology: General, 113, 372-393.

Rosenbaum, D. A., \& Kornblum, S. (1982). A priming method for investigating the selection of motor responses. Acta Psychologica, 51, 223-243.

STERNBERG, S. (1969). Memory scanning: Mental processes revealed by reaction-time experiments. American Scientist, 57, 421-457.

(Manuscript received September 2, 1986; revision accepted for publication July $6,1987$. 